\title{
Ordered Group Interference Suppression for V-BLAST
}

\author{
Jia Geng, Chenghao Guo \\ Science and Technology on Information Systems Engineering Laboratory \\ Nanjing, China \\ gengjia_cn@163.com
}

\begin{abstract}
As an efficient approach to increase data rate in wireless communication, vertical Bell laboratories layered space-time (V-BLAST) has been widely studied. In this paper an improved V-BLAST decoding algorithm, ordered group interference suppression (O-GIS), is presented, which reduces the error rate by calculating the norms of sub-channels and adjusting the decoding order of antenna groups. Then O-GIS is introduced to optimize the maximum likelihood ordered serial interference cancellation (ML-OSIC) algorithm for $\mathrm{V}$ BLAST, resulting in improved decoding performance at the expense of acceptable computation complexity.
\end{abstract}

Keywords-component; V-BLAST; decoding; orderd; group interference suppression

\section{INTRODUCTION}

Multi-input Multi-output (MIMO) [1, 2] is a key technique for the $4^{\text {th }}$ generation mobile communication. Vertical Bell laboratories layered space-time (V-BLAST) [3, $4]$ is an important kind of coding scheme of MIMO. The research hotspot of V-BLAST is decoding. Currently the decoding algorithms could be classified into four types, i.e. maximum likelihood (ML) [5], linear detection [6], interference cancellation (IC) [7], and other compound algorithms [8]. Among them, IC algorithms are widely accepted, because their performance and complexity could be balanced. Reference [9] proposed an IC algorithm named group interference suppression (GIS), which is optimized for the case when the number of transmit antennas is large. In this paper, we introduce the idea of "ordering" to GIS, and get a new algorithm called ordered GIS (O-GIS) with better performance. Reference [10] proposed a maximum likelihood ordered serial interference cancellation (ML-OSIC) algorithm for V-BLAST. If we also combine O-GIS with ML-OSIC, further improvement could be obtained.

\section{MODEL OF GROUP INTERFERENCE SUPPRESSION}

Suppose there are $n_{\mathrm{T}}$ transmit antennas, $n_{\mathrm{R}}$ receive antennas, and $B$ input bits for each time slot in the system. Separate the transmit antennas into $q$ groups, denoted as $G_{i}$ $(\mathrm{i}=1,2, \cdots q)$. For each group, the number of transmit antennas is $n_{i}(i=1,2, \cdots, q)$, and satisfies $n_{1}+n_{2}+\cdots+n_{q}=n_{\mathrm{T}}$. For any $\mathrm{G}_{j}(1 \leq j \leq q)$, the number of input bits for each time slot is $B_{j}$. These $B_{j}$ bits are encoded by space-time encoder $E_{j}$, and transmitted on the $n_{j}$-th antenna. Here $B_{1}+B_{2}+\cdots+B_{q}=B$, and $\mathrm{E}_{j}$ could be different encoders, such as BLAST, spacetime trellis code (STTC), and space-time block code (STBC). Fig. 1 shows the block diagram of the transmitter.
When $n_{\mathrm{T}}$ is large, if decoding the bits on all the transmit antennas simultaneously, it will be a big computation effort. Multi-level decoding is used to reduce the computation complexity with sub-optimized performance. In original algorithm described in [9], $G_{1}$ is firstly decoded, $G_{2}$ is the second one to be decoded, then $\mathrm{G}_{3}$ is the third, and so on. Since only one group is decoded for each time, the number of transmit antennas in a group is much lower than $n_{\mathrm{T}}$, so the overall decoding complexity could be reduced. To be able to decode any $\mathrm{G}_{j}$ in receiver, the interference introduced by other groups must be removed. For groups which already have been decoded, IC algorithm is used. For un-decoded groups, GIS algorithm could be applied. How GIS works is illustrated as following.

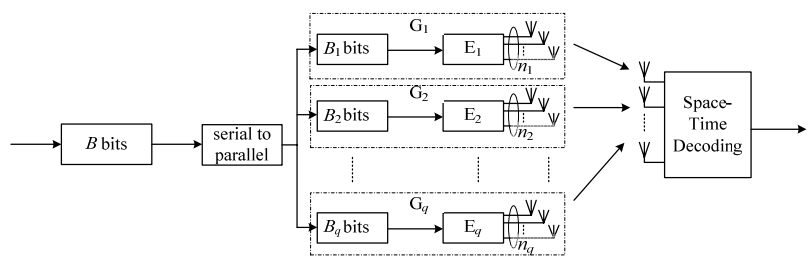

Figure 1. Figure1. block diagram of the transmitter is

Under flat Rayleigh fading channel, the model of BLAST

where

$$
\boldsymbol{r}=\boldsymbol{H} \boldsymbol{c}+\boldsymbol{n}
$$

$$
\begin{gathered}
\boldsymbol{r}=\left[\begin{array}{cccc}
r_{1}^{1} & r_{2}^{1} & \cdots & r_{T}^{1} \\
r_{1}^{2} & r_{2}^{2} & \cdots & r_{T}^{2} \\
\vdots & \vdots & \vdots & \vdots \\
r_{1}^{n_{\mathrm{R}}} & r_{2}^{n_{\mathrm{R}}} & \cdots & r_{T}^{n_{\mathrm{R}}}
\end{array}\right], \quad \boldsymbol{H}=\left[\begin{array}{cccc}
h_{1,1} & h_{1,2} & \cdots & h_{1, n_{\mathrm{T}}} \\
h_{2,1} & h_{2,2} & \cdots & h_{2, n_{\mathrm{T}}} \\
\vdots & \vdots & \vdots & \vdots \\
h_{n_{\mathrm{R}}, 1} & h_{n_{\mathrm{R}}, 2} & \cdots & h_{n_{\mathrm{R}}, n_{\mathrm{T}}}
\end{array}\right], \\
\boldsymbol{c}=\left[\begin{array}{ccccc}
c_{1}^{1} & c_{2}^{1} & \cdots & c_{T}^{1} \\
c_{1}^{2} & c_{2}^{2} & \cdots & c_{T}^{2} \\
\vdots & \vdots & \vdots & \vdots \\
c_{1}^{n_{\mathrm{T}}} & c_{2}^{n_{\mathrm{T}}} & \cdots & c_{T}^{n_{\mathrm{T}}}
\end{array}\right], \quad \boldsymbol{n}=\left[\begin{array}{cccc}
n_{1}^{1} & n_{2}^{1} & \cdots & n_{T}^{1} \\
n_{1}^{2} & n_{2}^{2} & \cdots & n_{T}^{2} \\
\vdots & \vdots & \vdots & \vdots \\
n_{1}^{n_{\mathrm{R}}} & n_{2}^{n_{\mathrm{R}}} & \cdots & n_{T}^{n_{\mathrm{R}}}
\end{array}\right] .
\end{gathered}
$$

$\boldsymbol{r}$ is receive matrix; $r_{t}^{j}$ is the received signal by antenna $j$ at time slot $t ; \boldsymbol{H}$ is channel matrix; $h_{j, i}$ is the channel coefficient from the transmit antenna $i$ to receive antenna $j$, which is complex Gaussian distributed with mean zero and variance 0.5 per dimension; $c$ is transmit data matrix; $c_{t}^{i}$ is modulated symbol sent from antenna $i$ at time slot $t ; \boldsymbol{n}$ is 
noise matrix; $n_{t}^{j}$ is AWGN noise on receive antenna $j$ at time slot $t$, which is complex Gaussian distributed with mean zero and variance $N_{0} / 2$ per dimension.

The first group to be decoded is $\mathrm{G}_{1}$, so $\boldsymbol{H}$ could be rewritten as

\section{$H=\left[\begin{array}{ll}H_{1} & H_{2}\end{array}\right]$}

where $\boldsymbol{H}_{1}$ is the matrix composed by the first $n_{1}$ columns of $\boldsymbol{H}$, and $\boldsymbol{H}_{2}$ is the matrix composed by the rest $n_{\mathrm{T}}-n_{1}$ columns of $\boldsymbol{H}$.

If applying singular value decomposition (SVD) to $\boldsymbol{H}_{2}$, we have

$$
\boldsymbol{H}_{2}=\boldsymbol{U} \boldsymbol{\Sigma} \boldsymbol{V}^{\mathrm{T}}
$$

where $\boldsymbol{U}$ and $\boldsymbol{V}$ are unitary matrixes, and $\boldsymbol{\Sigma}$ is diagonal matrix

Define

$$
\tilde{\boldsymbol{U}}=\left[\begin{array}{c}
(\boldsymbol{U})_{n_{\mathrm{T}}-n_{1}+1}^{\mathrm{H}} \\
(\boldsymbol{U})_{n_{\mathrm{T}}-n_{1}+2}^{\mathrm{H}} \\
\vdots \\
(\boldsymbol{U})_{n_{\mathrm{R}}}^{\mathrm{H}}
\end{array}\right]
$$

where $(\boldsymbol{U})_{i}$ is the $i$-th column of $\boldsymbol{U}$. Multiply (1) by $\tilde{\boldsymbol{U}}$, then

$$
\tilde{\boldsymbol{r}}=\tilde{\boldsymbol{H}} \boldsymbol{c}+\tilde{\boldsymbol{n}}
$$

where $\tilde{\boldsymbol{r}}=\tilde{\boldsymbol{U}} \boldsymbol{r}, \tilde{\boldsymbol{H}}=\tilde{\boldsymbol{U}} \boldsymbol{H}, \tilde{\boldsymbol{n}}=\tilde{\boldsymbol{U}} \boldsymbol{n}$. According to the definition of $\tilde{\boldsymbol{U}}, \tilde{\boldsymbol{U}} \boldsymbol{H}_{2}=\mathbf{0}$ could be derived, so we have $\tilde{\boldsymbol{H}}=\left[\begin{array}{ll}\tilde{U} \boldsymbol{H}_{1} & 0\end{array}\right]$.

Define $\tilde{\boldsymbol{c}}$ is the matrix composed by the first $n_{1}$ rows of matrix $\boldsymbol{c}$, and $\tilde{\boldsymbol{H}}^{\prime}=\left[(\tilde{\boldsymbol{H}})_{1}(\tilde{\boldsymbol{H}})_{2} \cdots(\tilde{\boldsymbol{H}})_{n_{1}}\right] .(\tilde{\boldsymbol{H}})_{i}$ is the $i$-th column in $\tilde{\boldsymbol{H}}$. So (5) can be rewritten as

$$
\tilde{\boldsymbol{r}}=\tilde{\boldsymbol{H}}^{\prime} \tilde{\boldsymbol{c}}+\tilde{\boldsymbol{n}}
$$

Equation (6) shows the interferences from other groups have been removed. This is called group interference suppression (GIS) in [9]. According to (6), if the decoding algorithm is applied to $E_{1}$, then we can get the estimation of $B_{1} T$ bits during $T$ slots for $G_{1}$, denoted as $\hat{\boldsymbol{c}}$. If these $B_{1} T$ bits are correctly decoded, then their information could be removed from received signal by

$$
\boldsymbol{r}^{\prime}=\boldsymbol{r}-\boldsymbol{H}_{1} \hat{\boldsymbol{c}}
$$

Define $\boldsymbol{c}^{\prime}$ is the matrix composed by the rest $n_{\mathrm{T}}-n_{1}$ rows of $c$. So we have the new system

$$
\boldsymbol{r}^{\prime}=\boldsymbol{H}_{2} \boldsymbol{c}^{\prime}+\boldsymbol{n}
$$

Repeat above steps, then $\mathrm{G}_{2}, \mathrm{G}_{3}, \cdots, \mathrm{G}_{q}$ could be decoded one by one.

\section{ORDERED GROUP INTERFERENCE SUPPRESSION}

Original GIS algorithm decodes the groups according to their natural order, i.e. $G_{1}, G_{2}, \ldots$, and the last $G_{q}$. If the group decoding order is tuned, then it's possible that the performance is optimized.

Suppose the first one to be decoded is $k$-th group, then $\boldsymbol{H}$ could be rewritten as

$$
\boldsymbol{H}=\left[\begin{array}{lll}
\boldsymbol{H}_{1}^{(k)} & \boldsymbol{H}_{2}^{(k)} & \boldsymbol{H}_{3}^{(k)}
\end{array}\right]
$$

where

$$
\begin{aligned}
& \boldsymbol{H}_{1}^{(k)}=\left[\begin{array}{cccc}
h_{1,1} & h_{1,2} & \cdots & h_{1, n_{1}+n_{2}+\cdot+n_{k-1}} \\
h_{2,1} & h_{2,2} & \cdots & h_{2, n_{1}+n_{2}+\cdot \cdot+n_{k-1}} \\
\vdots & \vdots & \vdots & \vdots \\
h_{n_{R}, 1} & h_{n_{R}, 2} & \cdots & h_{n_{R}, n_{1}+n_{2}+\cdot+n_{k-1}}
\end{array}\right] \\
& \boldsymbol{H}_{2}^{(k)}=\left[\begin{array}{cccc}
h_{1, n_{1}+n_{2}+\cdots+n_{k-1}+1} & h_{1, n_{1}+n_{2}+\cdots+n_{k-1}+2} & \cdots & h_{1, n_{1}+n_{2}+\cdots+n_{k-1}+n_{k}} \\
h_{2, n_{1}+n_{2}+\cdots+n_{k-1}+1} & h_{2, n_{1}+n_{2}+\cdots+n_{k-1}+2} & \cdots & h_{2, n_{1}+n_{2}+\cdots+n_{k-1}+n_{k}} \\
\vdots & \vdots & \vdots & \vdots \\
h_{n_{R}, n_{1}+n_{2}+\cdots+n_{k-1}+1} & h_{n_{R}, n_{1}+n_{2}+\cdots+n_{k-1}+2} & \cdots & h_{n_{R}, n_{1}+n_{2}+\cdots+n_{k-1}+n_{k}}
\end{array}\right] \text {, } \\
& \boldsymbol{H}_{3}^{(k)}=\left[\begin{array}{cccc}
h_{1, n_{1}+n_{2}+\cdots+n_{k}+1} & h_{1, n_{1}+n_{2}+\cdots+n_{k}+2} & \cdots & h_{1, n_{\mathrm{T}}} \\
h_{2, n_{1}+n_{2}+\cdots+n_{k}+1} & h_{2, n_{1}+n_{2}+\cdots+n_{k}+2} & \cdots & h_{2, n_{\mathrm{T}}} \\
\vdots & \vdots & \vdots & \vdots \\
h_{n_{\mathrm{R}}, n_{1}+n_{2}+\cdots+n_{k}+1} & h_{n_{\mathrm{R}}, n_{1}+n_{2}+\cdots+n_{k}+2} & \cdots & h_{n_{\mathrm{R}}, n_{\mathrm{T}}}
\end{array}\right] .
\end{aligned}
$$

Define $\boldsymbol{H}_{13}^{(k)}=\left[\boldsymbol{H}_{1}^{(k)} \boldsymbol{H}_{3}^{(k)}\right]$, if $1<k<q$; if $k=1$, then $\boldsymbol{H}_{1}^{(k)}$ is an empty matrix, $\boldsymbol{H}_{2}^{(k)}$ is the matrix composed by the first $n_{1}$ columns of $\boldsymbol{H}$, and the definitions of $\boldsymbol{H}, \boldsymbol{H}_{3}^{(k)}, \boldsymbol{H}_{13}^{(k)}$ are not changed; if $k=q$, then $\boldsymbol{H}_{3}^{(k)}$ is an empty matrix, and the definitions of $\boldsymbol{H}, \boldsymbol{H}_{1}^{(k)}, \boldsymbol{H}_{2}^{(k)}, \boldsymbol{H}_{13}^{(k)}$ are not changed。

Apply SVD to $\boldsymbol{H}_{13}^{(k)}$, then

$$
\boldsymbol{H}_{13}^{(k)}=\boldsymbol{U}^{(k)} \boldsymbol{\Sigma}^{(k)}\left(\boldsymbol{V}^{(k)}\right)^{\mathrm{T}}
$$

where $\boldsymbol{U}^{(k)}, \boldsymbol{V}^{(k)}$ are unitary matrixes, and $\boldsymbol{\Sigma}^{(k)}$ is diagonal matrix.

Define

$$
\tilde{\boldsymbol{U}}^{(k)}=\left[\begin{array}{c}
\left(\boldsymbol{U}^{(k)}\right)_{n_{\mathrm{T}}-n_{k}+1}^{\mathrm{H}} \\
\left(\boldsymbol{U}^{(k)}\right)_{n_{\mathrm{T}}-n_{k}+2}^{\mathrm{H}} \\
\vdots \\
\left(\boldsymbol{U}^{(k)}\right)_{n_{\mathrm{R}}}^{\mathrm{H}}
\end{array}\right]
$$

where $\left(\boldsymbol{U}^{(k)}\right)_{i}$ is the $i$-th column of $\boldsymbol{U}^{(k)}$.

Multiply (1) by $\tilde{\boldsymbol{U}}^{(k)}$

$$
\tilde{\boldsymbol{U}}^{(k)} \boldsymbol{r}=\tilde{\boldsymbol{U}}^{(k)} \boldsymbol{H} \boldsymbol{c}+\tilde{\boldsymbol{U}}^{(k)} \boldsymbol{n}
$$

Then 


$$
\tilde{\boldsymbol{r}}^{(k)}=\tilde{\boldsymbol{H}}^{(k)} \boldsymbol{c}+\tilde{\boldsymbol{n}}^{(k)}
$$

where $\tilde{\boldsymbol{r}}^{(k)}=\tilde{\boldsymbol{U}}^{(k)} \boldsymbol{r}, \tilde{\boldsymbol{H}}^{(k)}=\tilde{\boldsymbol{U}}^{(k)} \boldsymbol{H}, \tilde{\boldsymbol{n}}^{(k)}=\tilde{\boldsymbol{U}}^{(k)} \boldsymbol{n}$.

Since $\tilde{\boldsymbol{U}}^{(k)}\left[\boldsymbol{H}_{1}^{(k)} \boldsymbol{H}_{3}^{(k)}\right]=\mathbf{0}$, we have

$$
\tilde{\boldsymbol{H}}^{(k)}=\left[\begin{array}{lll}
\mathbf{0} & \tilde{\boldsymbol{U}}^{(k)} \boldsymbol{H}_{2}^{(k)} & \mathbf{0}
\end{array}\right]
$$

Define

$$
\tilde{\boldsymbol{c}}^{(k)}=\left[\begin{array}{cccc}
c_{1}^{n_{1}+n_{2}+n_{k-1}+1} & c_{2}^{n_{1}+n_{2}+n_{k-1}+1} & \cdots & c_{T}^{n_{1}+n_{2}+n_{k-1}+1} \\
c_{1}^{n_{1}+n_{2}+n_{k-1}+2} & c_{2}^{n_{1}+n_{2}+n_{k-1}+2} & \cdots & c_{T}^{n_{1}+n_{2}+n_{k-1}+2} \\
\vdots & \vdots & \vdots & \vdots \\
c_{1}^{n_{1}+n_{2}+n_{k-1}+n_{k}} & c_{2}^{n_{1}+n_{2}+n_{k-1}+n_{k}} & \cdots & c_{T}^{n_{1}+n_{2}+n_{k-1}+n_{k}}
\end{array}\right]
$$

And

$$
\tilde{\boldsymbol{H}}^{\prime(k)}=\tilde{\boldsymbol{U}}^{(k)} \boldsymbol{H}_{2}^{(k)}
$$

So (13) could be rewritten as

$$
\tilde{\boldsymbol{r}}^{(k)}=\tilde{\boldsymbol{H}}^{(k)} \tilde{\boldsymbol{c}}^{(k)}+\tilde{\boldsymbol{n}}^{(k)}
$$

According to [11], the probability of transmitting $\tilde{\boldsymbol{c}}^{(k)}$ and deciding in favor of $\tilde{\boldsymbol{e}}^{(k)}$ satisfies

$$
P\left(\tilde{\boldsymbol{c}}^{(k)}, \tilde{\boldsymbol{e}}^{(k)} \mid \tilde{\boldsymbol{H}}^{\prime(k)}\right) \leq \exp \left(-d^{2}\left(\tilde{\boldsymbol{c}}^{(k)}, \tilde{\boldsymbol{e}}^{(k)}\right) \frac{E_{\mathrm{s}}}{4 N_{0}}\right)
$$

where $E_{\mathrm{s}}$ is the average energy of each transmitted symbol, $N_{0}$ is the power of noise, and

$$
d^{2}\left(\tilde{\boldsymbol{c}}^{(k)}, \tilde{\boldsymbol{e}}^{(k)}\right)=\left\|\tilde{\boldsymbol{H}}^{(k)}\left(\tilde{\boldsymbol{c}}^{(k)}-\tilde{\boldsymbol{e}}^{(k)}\right)\right\|^{2}
$$

Since Frobenius norm is consistent, so we have

$$
d^{2}\left(\tilde{\boldsymbol{c}}^{(k)}, \tilde{\boldsymbol{e}}^{(k)}\right) \leq\left\|\tilde{\boldsymbol{H}}^{\prime(k)}\right\|^{2} \cdot\left\|\tilde{\boldsymbol{c}}^{(k)}-\tilde{\boldsymbol{e}}^{(k)}\right\|^{2}
$$

According to (17) and (19), the probability becomes smaller, if $\left\|\tilde{\boldsymbol{H}}^{\prime(k)}\right\|^{2}$ gets larger. Therefore the group $\mathrm{G}_{\hat{j}}$, which is chose to be the first decoded group, should satisfy

$$
\hat{j}=\underset{1 \leq j \leq q}{\arg \max }\left\|\tilde{\boldsymbol{H}}^{\prime(j)}\right\|
$$

After $\mathrm{G}_{\hat{j}}$ is decoded, cancel its interference from the received signal. Then follow the rules above to choose another group to decode, and cancel its interference after it is decoded. Iterate in this way until all groups are decoded.

From above we can see, in our algorithm the order of decoding is decided by the order of norms. So we name our algorithm ordered GIS, and ordering is the difference between our proposal and original GIS.

Simulation was done with the following condition. Suppose $n_{\mathrm{T}}$ is 4 , transmit antennas are divided into 2 groups, and each group has 2 antennas encoded with STBC [12]. $n_{\mathrm{R}}$ is 4, modulation is BPSK, channel model has described previously. Performance result is shown in Fig. 1. In the figure, when bit error rate (BER) is lower than $10^{-3}$, our algorithm has more than $2 \mathrm{~dB}$ gain。

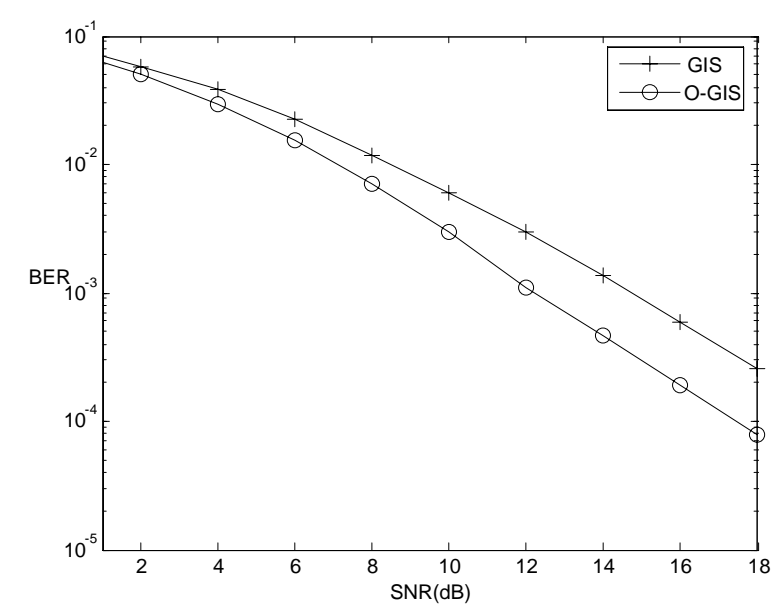

Fig. 1 Performance comparison of our O-GIS and original GIS

\section{ML-OSIC DECODING BASED ON OGIS}

Reference [10] proposed an improved V-BLAST decoding algorithm ML-OSIC. When decoding V-BLAST with OSIC, the first decoded layer has the minimal diversity, so the decoding of this layer is most unreliable. Once a detecting error occurs, the mistake will propagate to other layers, so it is the bottom-neck of decoding. According to the idea of GIS, transmit antennas are divided into 2 groups. Group one has fewer antennas. Denote its number of antennas is $p$, and usually $p$ is 2 . The rest antennas compose group 2. During detection, zero force is applied to group 2 firstly. Then decode group one with ML, followed by IC, and decode group two with OSIC. Since ML has high accuracy, the mistake propagation caused by the wrong first layer decoding could be reduced, and the overall reliability will be improved. Above is how original ML-OSIC works. [10] hasn't studied which antennas should compose group one. It only simply chooses the last $p$ antennas in nature order for group one.

However different antennas correspond to different channel gains, so different group one composition schemes have different error rates of decoding. Then here comes our improved algorithm. Collect all the possible group one composition schemes, totally $C_{n_{\mathrm{T}}}^{p}$ schemes. For each of schemes, e.g. the $i$-th scheme $\left(1 \leq i \leq C_{n_{\mathrm{T}}}^{p}\right)$, adjust the column order of channel matrix $\boldsymbol{H}$. Put the columns in group one to the left of $\boldsymbol{H}$, and keep other columns order unchanged. Calculate $\left.\left\|\tilde{\boldsymbol{H}}^{\prime(k)}\right\|^{2}\right|_{k=1}$ with the method described in O-GIS algorithm. This value has dependence on $i$, so it could be rewritten as $\left\|\tilde{\boldsymbol{H}}_{(i)}^{\prime(1)}\right\|^{2}$. Figure out $\hat{i}$, which satisfies

$$
\hat{i}=\underset{1 \leq i \leq C_{n_{\mathrm{T}}}^{p}}{\arg \max }\left\|\tilde{\boldsymbol{H}}_{(i)}^{(1)}\right\|^{2}
$$


then the $\hat{i}$-th scheme is the best. Once the scheme is lock down, the rest procedure of decoding is almost same as MLOSIC. Compared with ML-OSIC, our proposal needs extra $C_{n_{\mathrm{T}}}^{p}$ trials of group one composition schemes. The amount of computation is increased, but it's still acceptable when $n_{\mathrm{T}}$ is not very large. For current systems in practice, $n_{\mathrm{T}}$ does not exceed 4 generally.

For O-GIS proposed in last section, $\left\|\tilde{\boldsymbol{H}}^{(k)}\right\|^{2}$ is calculated after group division has been done, and the decision of group decoding order is based on $\left\|\tilde{\boldsymbol{H}}^{(k)}\right\|^{2}$. In this section, $\left\|\tilde{\boldsymbol{H}}_{(i)}^{\prime(1)}\right\|^{2}$ is calculated in the similar way as OGIS, but it is used for the selection of group one composition scheme, which is the difference from last section. The similarity is that, channel matrixes are ordered according to the norms, and error rate of early decoded groups could be reduced.

Suppose $n_{\mathrm{T}}$ and $n_{\mathrm{R}}$ are both 4, BPSK modulation. OSIC doesn't specify which algorithm should be used for IC. Considering minimal mean square error (MMSE) is widely used, so in our simulation, both ML-OSIC and our OGIS based ML-OSIC algorithm adopt MMSE. Simulation result is shown in Fig. 2. Our algorithm has $1 \mathrm{~dB}$ performance advantage.

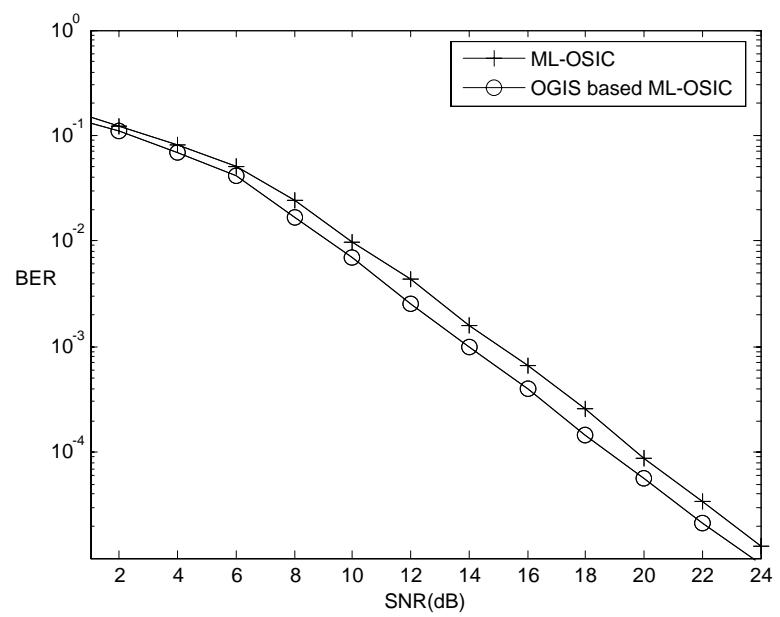

Fig. 2 Performance comparison of our algorithm and ML-OSIC

\section{CONCLUSION}

Our proposed O-GIS has $2 \mathrm{~dB}$ advantage over original GIS. And if O-GIS is applied to ML-OSIC, $1 \mathrm{~dB}$ gain could be obtained. The improvements are both caused by ordering the norms of channel matrixes, which decreases the error detection probability of first decoded groups, and reduces the error propagation to other groups.

\section{REFERENCES}

[1] Jan Mietzner, Robert Schober, Lutz Lampe, Wolfgang H.Gerstacker, Peter A. Hoeher, "Multiple-antenna techniques for wireless communications-a comprehensive literature survey," IEEE. Communications Surveys \& Tutorials.2009, 11(2): 87-105.

[2] Zhao Shao, MIMO System and STC: The Theory and Practice of Diversity. Beijing: Publishing House of Electronics Industry, 2013

[3] G.J. Foschini, "Layered space-time architecture for wireless communication in a fading environment when using multiple antennas," Bell laboratories Technical Journal, vol.1, no.2, pp.41-59, Autumn, 1996.

[4] P.W. Wolniansky, G.J. Foschini, G.D. Golden, "V-BLAST: an architecture for realizing very high date rates over the rich-scattering wireless channel," International Symposium on Signals, Systems, and Electronics 1998, pp.295 - 300, Sept, 1998.

[5] R.Van Nee,A.Van Zelst, and G. Awater, "Maximum likehood decoding in a space division multiplexing system," in Proc. vehicular Technology Conf.(Spring), 2000, 1:6-10.

[6] J.Benesty,Y.Huang, and J.Chen, "A fast recursive algorihm for optimum sequential signal detection in a BLAST system," IEEE Transactions on Signal Processing, July 2003, vol.51, no.7, pp.17221730 .

[7] Zhendong Luo, Ming Zhao, Siyang Liu, "Generalized parallel interference cancellation with near-optimal detection performance," IEEE transactions on Signal Processing , 2008, 56(1) : 304-312

[8] Jianping Zheng, Baoming Bai, Xinmei Wang, "Combining sphere decoding with M-algorithm," Proceedings of IEEE information theory workshop, 2006.

[9] V. Tarokh, A. Naguib, N. Seshadri, "Combined array processing and space-time coding," IEEE Trans. Inform. Theory, vol.45, no.4, pp.1121-1128, 1999.

[10] W-J. Choi, R. Negi, J.M. Cioffi, "Combined ML and DFE decoding for the V-BLAST system," IEEE ICC2002, vol.3, pp.1243-1248, June 2002.

[11] V. Tarokh, N. Seshadri, A.R. Calderbank, "Space-time codes for high data rate wireless communication: performance criterion and code construction," IEEE Trans. Inform. Theory, vol.44, no.2, pp.744-765, 1998.

[12] S.M. Alamouti, "A simple transmitter diversity scheme for wireless communication", IEEE Journal on Selected Area Comm., vol.16, no.10, pp.1451-1458, 1998 . 